\title{
EDITORIAL
}

\section{PASSIVE IMMUNOTHERAPY FOR ALZHEIMER'S DISEASE: WHAT HAVE WE LEARNED, AND WHERE ARE WE HEADED?}

\author{
P.S. AISEN ${ }^{1}$, B. VELLAS ${ }^{2}$ \\ 1. University of California in San-Diego, Alzheimer's Disease Collaborative Study: USA; 2. Gerontopole, Univeristy of Toulouse, UMR INSERM 1027 University Toulouse 3 , \\ CHU Toulouse, France
}

From the development of the first transgenic mouse model of brain amyloidosis (1), and the remarkable report on the dramatic effect of active immunization against aggregated amyloid peptide on this model (2), anti-amyloid immunotherapy has been the leading strategy for diseasemodifying drug development. But progress has been devastatingly slow. Development of the active vaccine for human $\mathrm{AD}$ was halted because of meningoencephalitis in a small percentage of treated individuals (3). While efforts turned to the development of safer active vaccines, using short sequence antigens to minimize toxicity mediated by cellular immunity, many companies sought development of passive immunotherapy. This approach affords much greater control- a monoclonal antibody, free of risk of cellular immune response, with controllable dose and frequency. Four monoclonal antiamyloid antibodies have advanced into mid- and late-phase trials.

Bapineuzumab is a humanized monoclonal antibody directed against the N-terminal sequence of the amyloid peptide (4). It is based on the most prevalent type of antibody induced by the failed active vaccination program. N-terminal epitopes are displayed in aggregated forms of amyloid, so these antibodies recognize aggregates including deposited fibrils. Animal studies indicate brain amyloid reduction, and suggest enhanced microglial phagocytosis as a mechanism. Bapineuzumab carries a risk of vasogenic edema and microhemmorhage (ARIA-E and ARIA-H), with risk related to drug dose and number of apolipoprotein E alleles (5). A phase II amyloid PET study indicated that bapineuzumab treatment reduces fibrillar amyloid in brain in individuals with AD (6). Topline results announced by the sponsors indicate that bapineuzumab treatment did not improve cognition or function in the pivotal trials in mild to moderate AD.

In contrast to bapineuzumab, solanezumab is a humanized monoclonal antibody that targets a mid-sequence amyloid peptide epitope (7). This antibody binds tightly to monomeric amyloid peptides, but not to aggregated or fibrillar forms. Solanezumab is also effective in reducing amyloid in transgenic mice. The proposed mechanism involves peripheral sequestration of monomeric amyloid peptide, reducing the central amyloid pool. Solanezumab does not carry the same risk of ARIA as bapineuzumab. Topline results from the recently completed pivotal trials in mild to moderate $\mathrm{AD}$ indicate that primary efficacy goals were not met, though pooled analyses suggest cognitive benefits.

Crenezumab also binds to a mid-sequence epitope, but differs from solenazumab in that it possesses an IgG4 (rather than $\operatorname{IgG} 1$ ) backbone, so that it triggers less cytokine production from microglia while maintaining phagocytosis towards amyloid (8). In vitro studies indicate that crenezumab binds to $\mathrm{A} \beta$ fibrils and oligomers, and to some extent monomers. Crenezumab treatment reduces brain amyloid plaque burden in transgenic mice. Phase 1 studies suggest that crenezumab may not cause vasogenic edema, though sample sizes were small. Crenezumab has been selected for the coming trial in familial autosomal dominant AD conducted by the Alzheimer's Prevention Initiative group (9).

Gantenerumab is a fully human monoclonal antibody that binds to both N-terminus and mid-sequence epitopes, and shows high affinity for fibrils. Like bapineuzumab, early studies demonstrated that treatment reduced brain amyloid as indicated by PET imaging, supporting target engagement (10). Trials in mild to moderate $\mathrm{AD}$ and prodromal $\mathrm{AD}$ are under way.

SAR228810 is a humanized antibody that recognizes a particular conformational epitope that allows it to bind specifically protofibrils and fibrils. A phase 1 single and multiple dose administration clinical study in patients with Alzheimers Disease is ongoing.

Ongoing analyses of the data from the two most advanced monoclonal antibody programs will address critical issues beyond the primary efficacy results. To optimize the development of disease-modifiers, careful attention must be directed to lessons from these studies. What characteristics of antibodies (eg, epitope specificity, tightness of binding, inflammatory potential) relate to biomarker, cognitive and adverse effects? How robust is reduction in amyloid PET signal? What is the impact on biomarkers of AD neurodegeneration such as cerebrospinal fluid tau and p-tau? How does immunotherapy alter regional atrophy rates as indicated by volumetric magnetic resonance imaging? Is there evidence relating bioomarker effects to impact on cognition? Which cognitive assessments are most sensitive to immunotherapy effects? How do subject characteristics such as disease severity and genotype influence immunotherapy effects? Does the timing of biomarker effects support their use 
in early futility analyses? How are adverse effects best minimized and managed? The details of trial design must be based on the best available answers to these questions. Precompetitive collaboration and data-sharing will accelerate progress.

Most industry and academic scientists believe that antiamyloid therapy will be most effective early in the disease process, before there is substantial irreversible neurodegeneration and synaptic failure. Several companies are now pursuing pre-dementia trials, using biomarkers to select individuals with mild cognitive impairment and biomarker evidence of AD. Several academic consortia will soon launch anti-amyloid trials in preclinical (asymptomatic) disease, in sporadic and genetically-determined populations; these groups are pooling data, ideas and resources to increase the likelihood of success.

The putative importance of stage of disease on therapeutic effect must be considered in planning further studies; an agent that has minimal or no clinical efficacy in AD dementia could prove enormously beneficial if administered at the preclinical stage of $\mathrm{AD}$ (11). We cannot afford to prematurely abandon anti-amyloid interventions. It is essential that we utilize the lessons of completed trials (12), but continue to pursue trial designs to reveal disease-modification at the earliest possible stage. Indeed, we should work toward sufficient understanding of the onset of AD neurobiology to support the design of primary prevention studies, aiming to control amyloid dysregulation with single or combination anti-amyloid therapy before there is measurable brain amyloid accumulation.

\section{References}

1. Hsiao K, Chapman P, Nilsen S, et al. Correlative memory deficits, ABeta elevation, and amyloid plaques in transgenic mice. Science 1996;274:99-102.

2. Schenk D, Barbour R, Dunn W, et al. Immunization with amyloid-beta attenuates Alzheimer-disease-like pathology in the PDAPP mouse. Nature 1999;400:173-177.

3. Schenk D. Amyloid-beta immunotherapy for Alzheimer's disease: the end of the beginning. Nature reviews Neuroscience 2002;3:824-828.

4. Salloway S, Sperling R, Gilman S, et al. A phase 2 multiple ascending dose trial of bapineuzumab in mild to moderate Alzheimer disease. Neurology 2009;73:20612070 .

5. Sperling R, Salloway S, Brooks DJ, et al. Amyloid-related imaging abnormalities in patients with Alzheimer's disease treated with bapineuzumab: a retrospective analysis. Lancet Neurol 2012;11:241-249.

6. Rinne JO, Brooks DJ, Rossor MN, et al. 11C-PiB PET assessment of change in fibrillar amyloid-beta load in patients with Alzheimer's disease treated with bapineuzumab: a phase 2, double-blind, placebo-controlled, ascending-dose study. Lancet Neurol 2010;9:363-372.

7. Farlow M, Arnold SE, van Dyck CH, et al. Safety and biomarker effects of solanezumab in patients with Alzheimer's disease. Alzheimers Dement 2012;8:261271.

8. Adolfsson O, Pihlgren M, Toni N, et al. An effector-reduced anti-beta-amyloid (Abeta) antibody with unique abeta binding properties promotes neuroprotection and glial engulfment of Abeta. The Journal of neuroscience : the official journal of the Society for Neuroscience 2012;32:9677-9689.

9. Colombia at the centre of preclinical AD research. Lancet Neurol 2012;11:567.

10. Ostrowitzki S, Deptula D, Thurfjell L, et al. Mechanism of amyloid removal in patients with Alzheimer disease treated with gantenerumab. Arch Neurol 2012;69:198-207.

11. Sperling RA, Jack CR, Jr., Aisen PS. Testing the right target and right drug at the right stage. Sci Transl Med 2011;3:111 cm133.

12. Vellas B, Hampel H, Rouge-Bugat ME, et al. Alzheimer's Disease Therapeutic Trials: EU/US Task Force Report on Recruitment, Retention, and Methodology. J Nutr Health Aging. 2012 Apr;16(4):339-45. 